\title{
Cognitive impairment in stroke patients five years post-stroke is associated with anxious and depressive symptoms in family members
}

\author{
Daniela Rohde ${ }^{1}$, Eva Gaynor ${ }^{2}$, Margaret Large ${ }^{3}$, Orla Conway ${ }^{1}$, David Williams ${ }^{1}$, \\ Kathleen Bennett ${ }^{1}$, Elizabeth Callaly ${ }^{4}$, Eamon Dolan ${ }^{5}$, Anne Hickey ${ }^{1}$ \\ ${ }^{1}$ Division of Population Health Sciences, Royal College of Surgeons in Ireland, Dublin, Ireland. \\ 2Department of Medicine, Royal College of Surgeons in Ireland, Dublin, Ireland. \\ ${ }^{3}$ Clinical Research Centre, Royal College of Surgeons in Ireland, Beaumont Hospital, Dublin, Ireland. \\ ${ }^{4}$ Mater Misericordiae University Hospital, Geriatric Medicine, Dublin, Ireland. \\ ${ }^{5}$ Connolly Hospital Blanchardstown, Geriatric Medicine, Dublin, Ireland.
}

\section{Background}

Family members frequently assume the primary responsibility for providing long-term care and support to patients with stroke, which can lead to emotional, financial, and social strains..$^{1,2}$ Caregivers of patients with stroke experience elevated levels of anxiety and depression, which can adversely affect stroke patient recovery. ${ }^{2,3}$ Psychological distress tends to decrease in the longer term poststroke, as family members adapt to their roles and stroke patients improve functionally. ${ }^{1,2}$ However, cognitive decline, which is common post-stroke and can progress indefinitely, ${ }^{4}$ significantly impacts levels of caregiver depression and anxiety. ${ }^{5}$ Many longer-term caregivers of stroke patients therefore continue to face unmet needs years after the initial stroke, highlighting the need for routine and repeated assessments of caregiver wellbeing. ${ }^{3}$

\section{Aim}

The aim of this study was to explore the association between cognitive impairment in stroke patients and anxious or depressive symptoms in family members five years post-stroke.

\section{Methods}

As part of a five-year follow-up of the Action on Rehabilitation and Secondary Prevention Interventions in Stroke (ASPIRE-S) cohort of stroke patients, family members completed a self-report questionnaire. 5,6 Symptoms of anxiety and depression were assessed using the Hospital Anxiety and Depression Scale - Anxiety subscale (HADS-A scores $\geq 8$ ), and the Center for Epidemiologic Studies Depression scale (CES-D scores $\geq 16$ ), respectively. Cognitive impairment in stroke survivors was assessed using the Informant Questionnaire on Cognitive Decline in the Elderly (IQCODE scores $\geq 3.3$ ).

\section{Results}

77 family members participated, of whom 71 completed the HADS-A, and 51 the CES-D. Family members were spouses/partners of stroke patients $(n=49,63.6 \%)$, adult children $(n=22,38.6 \%)$ or siblings $(n=6$, $7.8 \%)$. The majority was female $(n=62,80.5 \%)$ (Table 1$)$.

Depressive symptoms were evident in $25.5 \%$ of family members, with $19.7 \%$ reporting symptoms of anxiety. Twenty-two stroke patients $(29.0 \%)$ were identified as having evidence of cognitive impairment. Family members of stroke patients with cognitive impairment were significantly more likely to report symptoms of depression [ageadjusted OR (95\% Cl): $6.80(1.65,28.04)]$ or anxiety [age-adjusted OR $(95 \% \mathrm{Cl}): 5.99(1.62,22.15)]$ than family members of patients without evidence of cognitive impairment (Table 2).

Age $(M, S D)(n=74)$

Sex

\section{Conclusion}

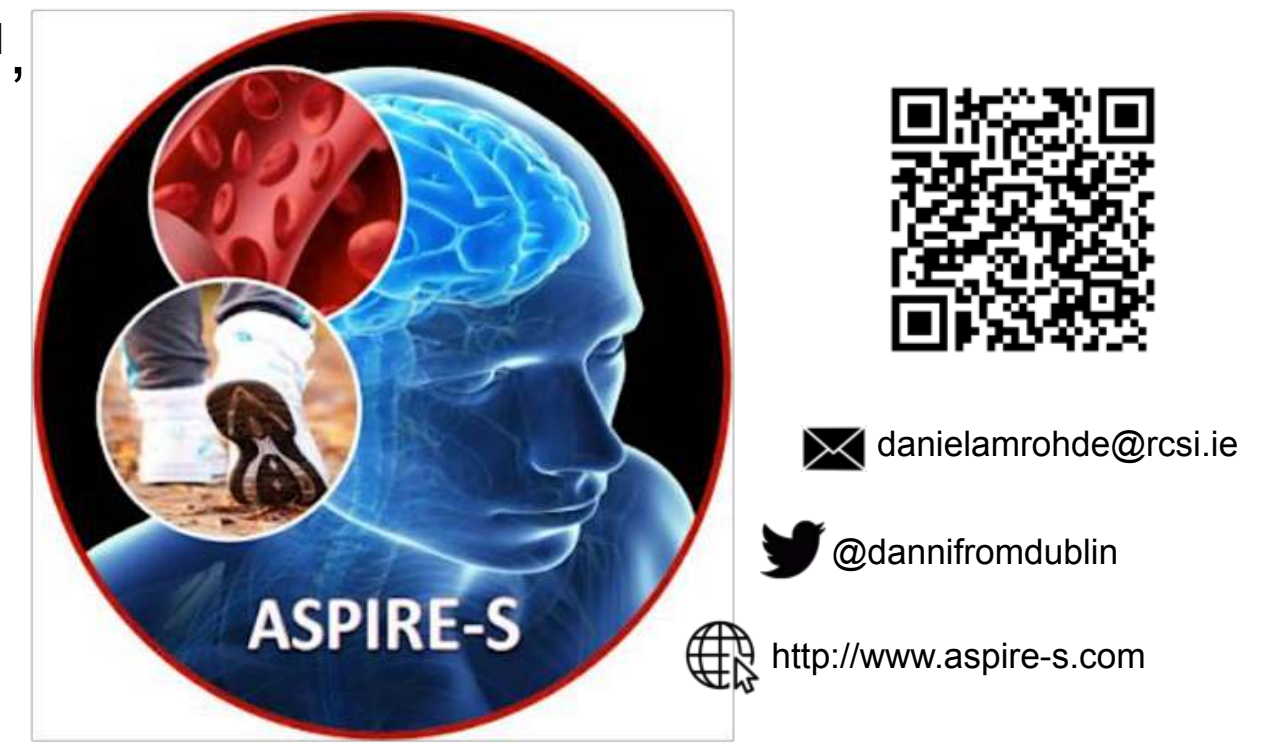

Table 1. Profile of family members of stroke patients 5 years post-stroke

$59.0(16.1)$

$15(19.5)$

\begin{tabular}{|llr|}
\cline { 2 - 3 } Sex & Female & $62(80.5)$ \\
\hline \multirow{2}{*}{ Relationship to stroke patient } & Spouse/partner & $49(63.6)$ \\
\cline { 2 - 3 } & Sibling & $6(7.8)$ \\
\cline { 2 - 3 } Symptoms of anxiety $(\mathrm{n}=71)$ & Adult child & $22(28.6)$ \\
\cline { 2 - 3 } & Anxious symptoms & $14(19.7)$ \\
\cline { 2 - 3 } Symptoms of depression $(\mathrm{n}=51)$ & None & $57(80.3)$ \\
\cline { 2 - 3 } Stroke patient & None & $13(25.5)$ \\
\hline cognitive impairment & Cognitive decline & $38(74.5)$ \\
\cline { 2 - 3 } & No decline & $54(29.0)$ \\
\hline
\end{tabular}

Stroke patient age (M, SD)

$68.8(12.3)$

Table 2. Age-adjusted ORs $(95 \% \mathrm{Cl})$ for logistic regression models of depressive and anxious symptoms of family members, based on stroke patient cognitive decline

\begin{tabular}{|c|c|c|}
\hline & Depressive symptoms & Anxious symptoms \\
\hline & \multicolumn{2}{|c|}{ aOR $(95 \% \mathrm{Cl})$} \\
\hline Cognitive decline & $6.80(1.65,28.04)^{\star \star}$ & $5.99(1.62,22.15)^{\star \star}$ \\
\hline Model & $\begin{aligned} X^{2}(2) & =8.69, p=.013, \\
\text { pseudo } R^{2} & =0.1533, n=49\end{aligned}$ & $\begin{array}{r}X^{2}(2)=10.53, p=.005, \\
\text { pseudo } R^{2}=0.1523, n=68\end{array}$ \\
\hline
\end{tabular}

Cognitive impairment in stroke patients continues to be associated with depressive and anxious symptoms in family members in the longer term. Family members play a key role in the care and rehabilitation of stroke patients; enhancing their psychological wellbeing and identifying ways to address these unmet needs is essential.

\footnotetext{
References

1. Pesantes MA, Brandt LR, Ipince A, et al. An exploration into caring for a stroke-survivor in Lima, Peru: Emotional impact, stress factors, coping mechanisms and unmet needs of informal caregivers. eNeurologicalSci. 2017:6:33-50.

2. Woodford J, Farrand P, Watkins ER, et al. "I Don't Believe in Leading a Life of My Own, I Lead His Life": A Qualitative Investigation of Difficulties Experienced by Informal Caregivers of Stroke Survivors Experiencing Depressive and Anxious Symptoms. Clin Gerontol. 2017:1-15.10.

3. Graf R, LeLaurin J, Schmitzberger M, et al. The stroke caregiving trajectory in relation to caregiver depressive symptoms, burden, and intervention outcomes. Top Stroke Rehabil. 2017:1-8.10.

4. Tang EY, Amiesimaka O, Harrison SL, et al. Longitudinal Effect of Stroke on Cognition: A Systematic Review. J Am Heart Assoc. 2018;7(2).

5. Atteih S, Mellon L, Hall P, et al. Implications of stroke for caregiver outcomes: findings from the ASPIRE-S study. Int J Stroke. 2015;10(6):918-23.

6. Rohde D, Williams D, Gaynor E, et al. Secondary prevention and cognitive function after stroke: a study protocol for a 5-year follow-up of the ASPIRE-S cohort. BMJ Open. 2017;7(3):e014819.
.
} 